\title{
Colonización cervicovaginal y rectal por estreptococos del grupo B en embarazadas mexicanas a término
}

\author{
Azucena L. Cabrera-Reyes ${ }^{1}$, Ma. del Carmen Martínez-García², Gerardo del C. Palacios-Saucedo², \\ Othón Rojas-Montes ${ }^{4}$, Adriana Cajero-Avelar ${ }^{5}$ y Fortino Solórzano-Santos²* \\ ${ }^{1}$ Departamento de Pediatría Hospital General de Zona 27, Hospital de Gineco-Obstetricia Tlatelolco, Instituto Mexicano del Seguro Social, Ciudad \\ de México; '2Unidad de Investigación de Enfermedades Infecciosas, Hospital Infantil de México Federico Gómez, Secretaría de Salud, Ciudad de \\ México; ${ }^{3}$ División de Investigación en Salud, UMAE Hospital de Especialidades N.․ 25, Instituto Mexicano del Seguro Social, Monterrey, N.L.; \\ ${ }^{4}$ Unidad de Investigación Médica en Enfermedades Infecciosas, UMAE Hospital de Pediatría Centro Médico Nacional SXXI, Instituto Mexicano del \\ Seguro Social, Ciudad de México; ${ }^{5}$ Departamento de Enfermedades Infecciosas Pediátricas, Hospital Centenario Miguel Hidalgo, Secretaría de \\ Salud, Aguascalientes, Ags. México
}

\section{Resumen}

Antecedentes: Una mujer embarazada colonizada por estreptococo del grupo B por vía rectal o vaginal puede infectar a su recién nacido. Pacientes y métodos: Estudio prospectivo, transversal y analítico, durante 24 meses, en embarazadas. Se incluyeron aquellas en trabajo de parto con $\geq 36$ semanas de gestación. El embarazo se clasificó como normal o de alto riesgo. Se analizaron los principales factores de riesgo de las embarazadas. Se tomaron muestras rectales y vaginales, se colocaron en caldo Todd-Hewitt y posteriormente se inocularon en agar sangre de carnero al 5\%. La identificación se realizó mediante pruebas bioquímicas y aglutinación con látex. Resultados: Se incluyeron 3,347 embarazadas, edad media $25.6 \pm 5.3$ años, 95.5\% con control prenatal; 2,213 (66\%) embarazo de riesgo normal y 1,370 (41\%) obtenidas por cesárea. La colonización global fue del 4.3\% (145/3,347), siendo mayor en el grupo de edad de 30 a 34 años (6.8\%). El serotipo I (58\%) fue el más frecuente. Conclusión: El porcentaje de colonización en esta población fue bajo. Un programa sistemático de cultivo cervicovaginal y rectal en mujeres embarazadas y el programa de profilaxis antimicrobiana intraparto son controvertidos en nuestra región.

PALABRAS CLAVE: Colonización cervicovaginal. Estreptococo del grupo B. Embarazo.

\section{Cervicovaginal and rectal colonization by group B Streptococcus in Mexican women with full-term pregnancies}

\section{Abstract}

Background: A pregnant woman rectally or vaginally colonized by group B Streptococcus can infect her newborn. Patients and methods: Prospective, cross-sectional, analytical 24-month study in pregnant women. Women in labor with $\geq 36$ weeks of gestation were included. Pregnancy was classified as normal or high-risk. Main risk factors of the pregnant women were analyzed. Rectal and vaginal samples were obtained, placed in Todd-Hewitt broth and subsequently inoculated in $5 \%$ sheep blood agar. Identification was carried out by biochemical tests and latex agglutination. Results: 3,347 pregnant women were included. Mean age was $25.6 \pm 5.3$ years, $95.5 \%$ received antenatal care; 2,213 (66 \%) had normal-risk pregnancies, and in 1,370 (41\%), delivery was by cesarean section. Overall colonization was $4.3 \%$ (145/3,347), and it was higher in the 30-34 years age group (6.8 \%). Serotype I (58 $\%)$ was the most common. Conclusion: The percentage of colonization in this population was low. A routine cervicovaginal and rectal culture program in pregnant women and the intrapartum antimicrobial prophylaxis program are controversial in our region.

KEY WORDS: Cervicovaginal colonization. Group B streptococcus. Pregnancy.

Correspondencia:

*Fortino Solórzano-Santos

E-mail: solorzanof056@gmail.com
Fecha de recepción: 26-07-2021

Fecha de aceptación: 12-08-2021

DOI: $10.24875 /$ GMM.21000466
Gac Med Mex. 2021;157:533-538

Disponible en PubMed

www.gacetamedicademexico.com

0016-3813/@ 2021 Academia Nacional de Medicina de México, A.C. Publicado por Permanyer. Este es un artículo open access bajo la licencia CC BY-NC-ND (http://creativecommons.org/licenses/by-nc-nd/4.0/). 
Streptococcus agalactiae o Streptococcus del grupo $B$ (SGB) es un coco grampositivo colonizador frecuente del tracto digestivo inferior.

Fry, en 1938, describió su capacidad como patógeno humano'. Desde la década de 1970 hubo un aumento de las infecciones perinatales por este microorganismo en países desarrollados y en la década de 1980 se consideró la causa más común de sepsis y meningitis neonatal ${ }^{2-5}$. EI SGB es uno de los principales agentes etiológicos en las infecciones perinatales, destacando la corioamnionitis, los abortos espontáneos y las infecciones posparto ${ }^{6}$.

Una madre con colonización genital o rectal por SGB podría transmitir este microorganismo a su recién nacido $(\mathrm{RN})$ por vía hematógena-transplacentaria, por vía ascendente o durante el paso por el canal cervicovaginal ${ }^{7}$. En los países desarrollados se han implementado diferentes medidas de prevención para prevenir la transmisión vertical, incluida la profilaxis antibiótica intraparto ${ }^{8,9}$. Para implementar estas medidas se requiere conocer la frecuencia de colonización materna por región específica para inferir el riesgo perinatal local.

La mayoría de los estudios sobre colonización e infección por SGB se han realizado en países desarrollados, en México la información es limitada en número de estudios y de regiones evaluadas ${ }^{10-15}$.

En este estudio se determinó la frecuencia de colonización cervicovaginal y rectal por SGB en una muestra de mujeres embarazadas atendidas en un hospital de ginecología-obstetricia de la Ciudad de México (CDMX).

\section{Pacientes y métodos}

\section{Diseño y pacientes}

Se realizó un estudio observacional, prospectivo, transversal y analítico durante 24 meses en mujeres embarazadas. Se incluyeron las embarazadas admitidas para atención del trabajo de parto (TP), se eligieron aquellas con $\geq 36$ semanas de embarazo y las de 35-36 semanas con TP activo y sin respuesta a la inhibición del TP. Se excluyeron las que habían recibido antibióticos (penicilinas, cefalosporinas, trimetoprima-sulfametoxazol y/o eritromicina) en el último trimestre del embarazo y aquellas con contraindicación médica (placenta previa, parto prematuro, desprendimiento de placenta). Los embarazos se clasificaron en riesgo normal o alto según la clasificación de Coopland ${ }^{16}$.

\section{Muestreo}

Un grupo de enfermeras capacitadas, en la primera revisión en la sala de partos o en el quirófano, colocó a cada paciente en posición ginecológica. Usando guantes estériles, se insertó en la vagina un hisopo de plástico de $15 \mathrm{~cm}$ con punta de algodón, se giró y se movió lateralmente; la muestra se colocó en un tubo con caldo Todd-Hewitt (TH) (Becton-Dickinson) con ácido nalidíxico $(15 \mu \mathrm{g} / \mathrm{ml})$ y gentamicina $(8 \mu \mathrm{g} / \mathrm{ml})$. En la misma posición se tomó una muestra de recto introduciendo $2 \mathrm{~cm}$ el hisopo en la ampolla rectal, girándolo sobre su propio eje y colocándolo en otro tubo con TH. Los tubos permanecieron en el área de parto a temperatura ambiente $\left(25^{\circ} \mathrm{C}\right)$ de 18 a 24 horas y todas las mañanas se trasladaron al laboratorio.

\section{Identificación bacteriana}

Los tubos de TH que presentaban turbidez se inocularon en placas de agar sangre de carnero al $5 \%$ (ASC5\%), los que no tenían turbidez se incubaron durante $48 \mathrm{~h}$ más, los que tuvieron turbidez se inocularon en ASC $5 \%$. Las placas se incubaron durante 24 horas a $37^{\circ} \mathrm{C}$, se aislaron colonias $\beta$-hemolíticas, grampositivo, prueba de catalasa negativa, hidrólisis de hipurato positiva y CAMP positiva. La identificación de antígenos de grupo y solubles para los serotipos I, II y III se realizó con prueba de aglutinación de látex (Pastorex B Streptococci ${ }^{\circledR}$, Sanofi Diagnostic Pasteur, Francia). En ausencia de aglutinación, el aislado se consideró no tipificable (NT).

El estudio fue aprobado por el Comité de Ética e Investigación del Hospital de Gineco-Obstetricia Tlatelolco (registro n. ${ }^{\circ}$ 98-701-0010). Se obtuvo el consentimiento informado por escrito de todas las participantes.

\section{Análisis estadístico}

Se utilizó estadística descriptiva para las características generales de la muestra. Se utilizó la prueba de chi cuadrada $\left(\chi^{2}\right)$ para comparar la prevalencia de colonización por SGB entre mujeres con embarazos habituales y de alto riesgo. Se consideró significativo un valor de $p<0.05$.

\section{Resultados}

Durante un periodo de 24 meses se incluyeron 3,347 mujeres embarazadas; 2,213 tenían embarazo 
de riesgo habitual (66\%) y 1,134 embarazo de alto riesgo (34\%). La edad media fue de $25.6 \pm 5.3$ años (rango: 14-43 años), el 95.5\% tenía control prenatal. Se realizó cesárea en 1,370 (41\%), siendo mayor en el grupo de embarazo de alto riesgo (62 vs. 32\%, $p<0.001)$. El $27.5 \%$ presentó infección urinaria, el $15.5 \%$ ruptura prematura de membranas (RPM), preeclampsia en el $10.8 \%$ y anemia en el $1.6 \%$. Hubo diferencias significativas en algunas características: edad, diabetes, preeclampsia, obesidad, fiebre y RPM $>12$ horas. El resto de características se presenta en la tabla 1.

\section{Colonización materna}

En las 3,347 embarazadas se obtuvieron cultivos vaginales y rectales, aislándose SGB en uno o ambos sitios en 145 , para una tasa de colonización del $4.3 \%$. Esta tasa fue mayor en mujeres entre 30 y 34 años $(6.8 \%)$ con una diferencia significativa con las menores de 19 años (3.6 vs. $6.8 \%, p=0.033$ ) y las menores de 25 y 30 años (Tabla 2). EI SGB se aisló solo de la vagina en $85 / 145(59 \%)$, del recto en $36 / 145(25 \%)$ y de ambos sitios en $24 / 145$ (17\%); esto corresponde a $60 / 145(42 \%)$ colonizados a nivel rectal y 109/145 (75\%) a nivel vaginal (Fig. 1). La colonización por SGB fue mayor en el grupo de embarazo de alto riesgo que en el grupo de riesgo habitual (5.9 vs. $3.4 \%, p=0.001$ ) y en mujeres casadas, con fiebre y antecedentes de infección urinaria. La frecuencia de RPM fue mayor en mujeres embarazadas no colonizadas. No hubo diferencia significativa en ninguna de las otras características evaluadas (Tabla 3).

\section{Serotipos}

El serotipo I (58\%) fue el más frecuente en ambos grupos. Aunque se observó una mayor identificación del serotipo III en el grupo de alto riesgo (24 vs. $21 \%$ ) con menor frecuencia de serotipo II (9 vs. $16 \%)$, no hubo diferencias significativas $(p=0.42)$ (Tabla 4).

\section{Discusión}

EI SGB se considera microbiota normal del intestino, la vagina y el recto, sin embargo, se le identifica como un patógeno importante del RN y la mujer embarazada. Aproximadamente una de cada cinco mujeres embarazadas en todo el mundo están colonizadas ${ }^{17}$, factor de riesgo conocido para enfermedad neonatal invasiva temprana ${ }^{18,19}$; la frecuencia varía según el sitio
Tabla 1. Características de 3,347 embarazadas atendidas en un hospital de gineco-obstetricia de la Ciudad de México

\begin{tabular}{|c|c|c|c|}
\hline & \multicolumn{2}{|c|}{ Riesgo del embarazo } & \multirow[t]{2}{*}{ p } \\
\hline & Habitual & Alto & \\
\hline $\begin{array}{l}\text { Edad (años) } \\
\text { (media } \pm \text { DE) }\end{array}$ & $24.9 \pm 4.9$ & $26.9 \pm 5.7$ & $0.0001^{\dagger}$ \\
\hline $\begin{array}{l}\text { Estado civil } \\
\text { Soltero } \\
\text { Casado }\end{array}$ & $\begin{array}{c}178(8 \%) \\
2,036(92 \%)\end{array}$ & $\begin{array}{c}57(5 \%) \\
1,076(95 \%)\end{array}$ & $0.003^{*}$ \\
\hline $\begin{array}{l}\text { Número de embarazos } \\
\leq 2 \\
\geq 3\end{array}$ & $\begin{array}{c}1,703(76.9 \%) \\
510(23 \%)\end{array}$ & $\begin{array}{l}775(68.3 \%) \\
357(31.4 \%)\end{array}$ & $0.0001^{*}$ \\
\hline Control prenatal & $2,110(95 \%)$ & $1,085(96 \%)$ & 0.28 \\
\hline Diabetes & $1(0.04 \%)$ & $7(0.6 \%)$ & $0.003^{*}$ \\
\hline Obesidad & $317(14 \%)$ & $467(41 \%)$ & $0.0001^{*}$ \\
\hline Preeclampsia & $46(2 \%)$ & $316(28 \%)$ & $0.0001^{*}$ \\
\hline Fiebre & $3(0.1 \%)$ & $11(1 \%)$ & $0.001^{*}$ \\
\hline Infección de vías urinarias & $8(0.4 \%)$ & $8(0.7 \%)$ & 0.13 \\
\hline Corioamnionitis & 0 & $4(0.4 \%)$ & $0.013^{*}$ \\
\hline $\mathrm{RPM}>12 \mathrm{~h}$ & $18(0.8 \%)$ & $60(5.3 \%)$ & $0.0001^{*}$ \\
\hline Cesárea & $681(32 \%)$ & $689(62 \%)$ & $0.0001^{*}$ \\
\hline Cultivo positivo SGB & 77 (3.47\%) & $68(5.99 \%)$ & $0.001^{*}$ \\
\hline Total & $2,213(66 \%)$ & $1,134(34 \%)$ & \\
\hline
\end{tabular}

*Prueba exacta de Fisher.

tPrueba t de Student.

DE: desviación estándar; RPM: ruptura prematura de membranas; SGB: Streptococcus del grupo $B$.

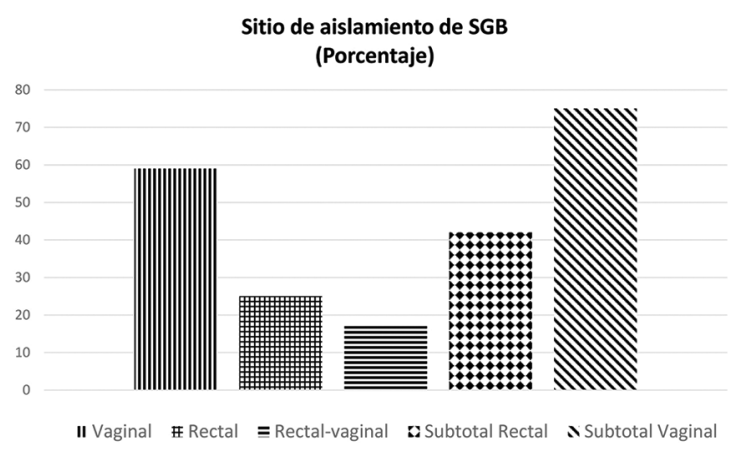

Figura 1. Sitio de aislamiento de Streptococcus del grupo B (SGB) en 145 gestantes atendidas en un hospital de gineco-obstetricia de la Ciudad de México.

colonizado, la edad gestacional, la ruta de nacimiento, así como las diferencias intrínsecas y la ubicación geográfica de las poblaciones estudiadas. 
Tabla 2. Colonización por Streptococcus del grupo B en embarazadas de un hospital de gineco-obstetricia de la Ciudad de México

\begin{tabular}{|c|c|c|c|}
\hline Edad (años) & $\begin{array}{c}\text { Mujeres embarazadas } \\
\text { colonizadas }\end{array}$ & $\begin{array}{c}\text { Total de mujeres } \\
\text { embarazadas }\end{array}$ & $p$ \\
\hline$\leq 19^{*}$ & $16(3.6 \%)$ & 442 & \\
\hline 20-24 & $33(3.1 \%)$ & 1,052 & 0.64 \\
\hline $25-29$ & $51(4.7 \%)$ & 1,093 & $0.007^{\dagger}$ \\
\hline 30-34 & 38 (6.8\%) & 556 & $0.033^{\ddagger}$ \\
\hline$>35$ & 7 (3.4\%) & 204 & $0.026^{\S}$ \\
\hline Total & 145 (4.3\%) & 3,347 & \\
\hline
\end{tabular}

No existe un estándar internacional establecido para el muestreo de colonización materna por SGB, lo que genera variaciones al comparar estudios de diferentes países. Estas variaciones pueden deberse al método y momento del muestreo, sitios anatómicos muestreados y técnicas de laboratorio utilizadas para transportar y cultivar muestras ${ }^{20,21}$.

En el presente estudio la frecuencia de colonización (4.3\%) fue menor que la reportada en el mundo (17.9\%, intervalo de confianza del 95\% [IC 95\%]: $16.2-19.7 \%)^{22}$, con un rango del $11.1 \%$ (IC 95\%: 6.8$15.3 \%$ ) en el sudeste asiático al $22.4 \%$ en África (IC 95\%: 18.1-26.7\%). Algunos países como Brasil (14\%), China (14\%), Nigeria (19.5\%) y Arabia Saudita (19\%) tienen una frecuencia alta ${ }^{22-27}$. En México también hay diferencias con estudios previos realizados: Collado, et al. en 1981 encontraron un 4\% de colonización ${ }^{10}$, sin embargo, en un centro de atención de tercer nivel en la CDMX se reportó un $10.3 \%$, en una población rural del sur del país encontraron un $8.6 \%{ }^{12}$ y en un hospital de segundo nivel en el occidente de México un $13 \%{ }^{13}$. La colonización en la población de alto riesgo del presente estudio fue menor a la encontrada en 1989 en la CDMX (6 vs. $10.3 \%)^{11}$. Quizá las diferencias el presente estudio podría explicarse por el tamaño de muestra y que el muestreo fue en mujeres embarazadas que estaban en TP, recién ingresadas y la mayoría con embarazos a término. La colonización por SGB fue mayor en mujeres con embarazo de alto riesgo, esta frecuencia aumenta a mayor edad, sin embargo, esta frecuencia es menor que la observada en otras poblaciones del mundo22-27.
Tabla 3. Características de embarazadas colonizadas y no colonizadas por Streptococcus del grupo B (SGB)

\begin{tabular}{|c|c|c|c|}
\hline & \multicolumn{2}{|c|}{ Cultivo SGB } & \multirow[t]{2}{*}{ p } \\
\hline & $\begin{array}{c}\text { Positivo } \\
\text { n (\%) }\end{array}$ & $\begin{array}{c}\text { Negativo } \\
\text { n (\%) }\end{array}$ & \\
\hline $\begin{array}{l}\text { Edad (años) } \\
\text { (Media } \pm D E \text { ) }\end{array}$ & $26.6 \pm 5$ & $25.5 \pm 5.2$ & $0.01^{*}$ \\
\hline $\begin{array}{l}\text { Estado civil } \\
\text { Soltero } \\
\text { Casado } \\
\text { Desconocido }\end{array}$ & $\begin{array}{c}5(3.4 \%) \\
138(95.1 \%) \\
2(1.4 \%)\end{array}$ & $\begin{array}{c}232(7.2 \%) \\
2,920(91.2 \%) \\
50(1.5 \%)\end{array}$ & $0.047^{\dagger}$ \\
\hline $\begin{array}{l}\text { Número de embarazos } \\
<3 \\
\geq 3\end{array}$ & $\begin{array}{c}105(72.4 \%) \\
40(27.6 \%)\end{array}$ & $\begin{array}{c}2,374(74.1 \%) \\
828(25.9 \%)\end{array}$ & $0.35^{\dagger}$ \\
\hline $\begin{array}{l}\text { Edad gestacional } \\
\text { (semanas) } \\
\leq 36 \\
\geq 37\end{array}$ & $\begin{array}{c}2(1.3 \%) \\
143(98.6 \%)\end{array}$ & $\begin{array}{c}132(4.1 \%) \\
3,070(95.9 \%)\end{array}$ & $0.06^{\ddagger}$ \\
\hline Obesidad & $40(28 \%)$ & 745 (23\%) & $0.13^{\dagger}$ \\
\hline Diabetes & 0 & $8(0.2 \%)$ & $0.7^{\dagger}$ \\
\hline RPM & $15(10.3 \%)$ & $505(16 \%)$ & $0.04^{+}$ \\
\hline Preeclampsia & $16(11 \%)$ & $346(11 \%)$ & $0.50^{+}$ \\
\hline Fiebre & $2(1.3 \%)$ & $11(0.3 \%)$ & $0.02^{\dagger}$ \\
\hline Infección urinaria reciente & $2(1.3 \%)$ & $14(0.4 \%)$ & $0.15^{+}$ \\
\hline Infección urinaria pasada & $50(34.4 \%)$ & $870(27 \%)$ & $0.03^{\dagger}$ \\
\hline Corioamnionitis & $1(0.7 \%)$ & $3(0.1 \%)$ & $0.16^{+}$ \\
\hline Total & $145(100 \%)$ & $3,202(100 \%)$ & \\
\hline
\end{tabular}

*Pruebat de Student.

TPrueba $\chi^{2}$

fPrueba exacta de Fisher.

DE: desviación estándar; RPM: ruptura prematura de membranas

Tabla 4. Serotipos de Streptococcus del grupo B (SGB) en mujeres con embarazo habitual y de alto riesgo

\begin{tabular}{|l|c|c|c|}
\multirow{2}{*}{ Serotipo } & \multicolumn{2}{|c|}{ Riesgo del embarazo } & \multirow{2}{*}{ Total } \\
\cline { 2 - 3 } & $\begin{array}{c}\text { Habitual } \\
\text { n (\%) }\end{array}$ & $\begin{array}{c}\text { Alto } \\
\text { n (\%) }\end{array}$ & \\
\hline I & $42(55 \%)$ & $42(62 \%)$ & $84(58 \%)$ \\
\hline II & $12(16 \%)$ & $6(9 \%)$ & $18(13 \%)$ \\
\hline III & $16(21 \%)$ & $17(25 \%)$ & $33(22 \%)$ \\
\hline NT & $7(9 \%)$ & $3(5 \%)$ & $10(7 \%)$ \\
\hline Cultivo SGB positivo & $77(100)$ & $68(100)$ & 145 \\
\hline
\end{tabular}

NT: no tipificable

Se ha sugerido que se deben realizar cultivos vaginales y rectales simultáneamente; en este estudio, el 
$25 \%$ de los aislamientos se obtuvieron solo del recto, por lo que, si solo se hubieran tomado cultivos vaginales, se hubiera escapado un porcentaje significativo de mujeres colonizadas. Lo anterior apoya el tomar muestras en ambos sitios ${ }^{28}$.

A nivel mundial, cinco serotipos de SGB (Ia, Ib, II, III y $\mathrm{V}$ ) representan el $98 \%$ de los serotipos predominantes ${ }^{17}$. En nuestro estudio predominó el serotipo I, como se informó anteriormente en México ${ }^{11-14}$ y en otras regiones del mundo ${ }^{17}$. El serotipo $V$ no se buscó en este estudio porque se consideró de muy baja frecuencia en México según estudios previos ${ }^{11-14}$.

Debido a la frecuencia de colonización e infecciones perinatales por SGB, en EE.UU. y otros países desarrollados se recomienda buscarlo de manera sistemática en todas las mujeres embarazadas entre las 35 y 37 semanas de gestación, y administrar profilaxis antibiótica a las mujeres colonizadas desde cuatro horas antes del parto. Esta estrategia ha logrado una disminución drástica en la incidencia de la enfermedad neonatal de aparición temprana, sin modificar la incidencia de infecciones de aparición tardía ${ }^{29}$. En otros países, esta política no se ha adoptado, por la falta de información sobre colonización y transmisión al RN. En los países subdesarrollados hay limitaciones para contar con datos epidemiológicos por la falta de pruebas fáciles, rápidas y de bajo costo. La realización de cultivos es una práctica costosa y que requiere mucho tiempo. Un escrutinio basado en el riesgo materno no ha ofrecido buenos resultados ${ }^{28}$.

En la población de mujeres mexicanas la colonización cervicovaginal y rectal por SGB es baja en comparación con otros países, por tanto, la realización de cultivos en todas las embarazadas no parece justificado en México, considerando el alto costo de esta práctica y la baja probabilidad de detectar mujeres colonizadas. Por ahora, la información disponible es insuficiente para establecer un programa de profilaxis antimicrobiana intraparto.

Una debilidad de este estudio es que solo se incluyeron mujeres embarazadas de la Ciudad de México, sin embargo, la información disponible de otros Estados del país sugiere que este bajo porcentaje de colonización es real ${ }^{12,13}$. Los factores que pueden estar condicionando esta baja tasa de colonización en la población mexicana aún se desconocen y, por lo tanto, constituyen un campo que requiere futuras investigaciones.

\section{Financiamiento}

Este estudio recibió financiamiento de las instituciones participantes del Instituto Mexicano del Seguro Social.

\section{Conflicto de intereses}

Los autores declaran no tener ningún conflicto de intereses

\section{Responsabilidades éticas}

Protección de personas y animales. Los autores declaran que los procedimientos seguidos se conformaron a las normas éticas del comité de experimentación humana responsable y de acuerdo con la Asociación Médica Mundial y la Declaración de Helsinki.

Confidencialidad de los datos. Los autores declaran que han seguido los protocolos de su centro de trabajo sobre la publicación de datos de pacientes.

Derecho a la privacidad y consentimiento informado. Los autores declaran que en este artículo no aparecen datos de pacientes.

\section{Bibliografía}

1. Fry RM. Fatal infections by hemolytic streptococcus group B. Lancet. 1938:1:199-201.

2. Eickhoff TC, Klein JO, Daly KA, Ingall D, Finland M. Neonatal sepsis and other infections due to group B beta-hemolytic Streptococci. N Engl J Med. 1964;271:1221-8.

3. Stoll BJ, Gordon T, Korones SB, Shankaran S, Tyson JE, Bauer CR. Early-onset sepsis in very low birth weight neonates: A report from the National Institute of Child Health and Human Development Neonatal Research Network. J Pediatr. 1996;129:73-80.

4. Baltimore RS, Huie SM, Meek JI, Schuchat A, O'Brien KL. Early-onset neonatal sepsis in the era of group $B$ Streptococcal prevention. Pediatrics 2001:108:1094-8.

5. Kalliola S, Vuopio-Varkila J, Takala AK, Eskola J. Neonatal group B streptococcal disease in Finland: a ten-year nationwide study. Pediatr Infect Dis J. 1999;18(9):806-10.

6. Patel K, Williams S, Guirguis G, Gittens-Williams L, Apuzzio J. Genita tract GBS and rate of histologic chorioamnionitis in patients with PPROM. J Matern Fetal Neonatal Med. 2018;31(19):2624-7.

7. Edmond KM, Kortsalioudaki C, Scott S, Schrag SJ, Zaidi AKM, Cousens S, et al. Group B streptococcal disease in infants aged younger than 3 months: systematic review and meta-analysis. Lancet. 2012;379:547-56.

8. Alhhazmi A, Hurteau D, Tyrrell GJ. Epidemiology of invasive group B streptococcal disease in Alberta, Canada, from 2003 to 2013. J Clin Microbiol. 2016;54(7):1774-81.

9. O'Sullivan CP, Lamagni T, Patel D, Efstratiou A, Cunney R, Meehan M, et al. Group B streptococcal disease in UK and Irish infants younger than 90 days, 2014-15: a prospective surveillance study. Lancet Infect Dis. 2019;19(1):83-90.

10. Collado M, Kretschmer RR, Becker I, Guzmán A, Gallardo L, Lepe CM. Colonization of Mexican pregnant women with group B streptococcus. $\mathrm{J}$ Infect Dis. 1981;143(1):134.

11. Solorzano-Santos F, Echaniz-Aviles G, Conde-Glez CJ, Calderon-Jaimes E, Arredondo-Garcia JL, Beltran-Zuniga M. Cervicovaginal infection with group B streptococci among pregnant Mexican women. J Infect Dis. 1989;159(5):1003-4. 
12. Ocampo-Torres M, Sánchez-Pérez HJ, Nazar-Beutelspacher A, Castro-Ramírez AE, Cordero-Ocampo B. Factors associated with Streptococcus group B colonization in pregnant women in Los Altos, Chiapas. Salud Pública Mex. 2000;42(5):413-21

13. Villaseñor-Sierra A, Morales-Velázquez P, Palacios-Saucedo G, Solórzano-Santos F. Prevalence of Streptococcus algalactiae serotype III in pregnant women. Ginecol Obstet Mex. 2004;72:103-8.

14. Palacios GC, González MN, Beltrán M, Arredondo لا J, Torres J, Solórzano F. Serotypes of 286 group B streptococci isolated from asymptomatic carriers and invasive disease cases in Mexico. Rev Latinoam Microbiol. 2005;47(1-2):21-4

15. Reyna-Figueroa J, Ortiz-lbarra FJ, Esteves-Jaramillo A, Casanova-Román G. Maternal B group Streptococcus colonization in Mexico: prevalence based on literature review. Ginecol Obstet Mex. 2007;75(7):399-403.

16. Coopland AT, Peddle LJ, Baskett TF, Rollwagen R, Simpson A, Parker E. A simplified antepartum high-risk pregnancy scoring form: statistical analysis of 5459 cases. Can Med Assoc J. 1977;116:999-1001.

17. Russell N, Seale AC, O'Driscoll M, O'Sullivan C, Bianchi-Jassir F, Gonzalez-Guarin J, et al. Maternal colonization with group B Streptococcus and serotype distribution worldwide: systematic review and meta-analyses. Clin Infect Dis. 2017;65(suppl 2):S100-111.

18. Dillon HC, Santosh K, Gray BM. Group B streptococcal carriage and disease: a 6-year prospective study. J Pediatr. 1987;110:31-6.

19. Alkalay AL, Brunell PA, Greenspon JS, Pomerance JJ. Management of neonates born to mothers with group B Streptococcus colonization J Perinatol. 1996;16:470-7.

20. Valkenburg-van den Berg AW, Houtman-Roelofsen RL, Oostvogel PM Dekker FW, Dörr PJ, Sprij AJ. Timing of group B Streptococcus screening in pregnancy: a systematic review. Gynecol Obstet Invest. 2010;69:174-83.
21. Badri MS, Zawaneh S, Cruz AC, Mantilla G, Baer H, Spellacy WN, et al. Rectal colonization with group B Streptococcus: relation to vaginal colonization of pregnant women. J Infect Dis. 1977;135:308-12.

22. Kwatra G, Cunnington MC, Merrall E, Adrian PV, Ip M, Klugman KP, et al. Prevalence of maternal colonization with group B Streptococcus: a systematic review and meta-analysis. Lancet Infect Dis. 2016; 16:1076-84.

23. Hickman ME, Rench MA, Ferrieri $P$, Baker CJ. Changing epidemiology of group B streptococcal colonization. Pediatrics. 1999;104:203-9.

24. Zhu Y, Huang J, Lin XZ, Chen C. Group B Streptococcus colonization in late pregnancy and invasive infection in neonates in China: A population-based 3-year study. Neonatology. 2019;115(4):301-9.

25. Musleh J, Al Qahtani N. Group B Streptococcus colonization among Saudi women during labor. Saudi J Med Med Sci. 2018;6(1):18-22.

26. Siqueira F, Ferreira EM, de Matos Calderon I, Dias A. Prevalence of colonization by group B streptococcus in pregnant patients in Taguatinga, Federal District, Brazil: a cross-sectional study. Arch Gynecol Obstet. 2019;299(3):703-11

27. Gizachew M, Tiruneh M, Moges F, Tessema B. Streptococcus agalactiae maternal colonization, antibiotic resistance and serotype profiles in Africa: a meta-analysis. Ann Clin Microbiol Antimicrob. 2019;18(1):14.

28. Khalil MR, Uldbjerg N, Thorsen PB, Møller JK. Risk-based approach versus culture-based screening for identification of group B streptococci among women in labor. Int J Gynaecol Obstet. 2019; 144(2):187-91.

29. Schrag SJ, Verani JR. Intrapartum antibiotic prophylaxis for the prevention of perinatal group B streptococcal disease: experience in the United States and implications for a potential group B streptococcal vaccine. Vaccine. 2013;31(Suppl 4):D20-6. 